\title{
Author Correction: Nanostructured laminar tungsten alloy with improved ductility by surface mechanical attrition treatment
}

\author{
Hong-Yan Guo 1,2,3, Min Xia ${ }^{2}$, Lap-Chung Chan ${ }^{3}$, Kun Wang ${ }^{4}$, Xiao-Xin Zhang ${ }^{2}$, Qing-ZhiYan², \\ Man-Chao $\mathrm{He}^{1}$, Jian $\mathrm{Lu}^{3,5}$ \& Chang-Chun Ge${ }^{2}$
}

Correction to: Scientific Reports https://doi.org/10.1038/s41598-017-01458-0, published online 02 May 2017

The original version of this Article omitted an affiliation for Jian Lu. The correct affiliations are listed below:

Department of Mechanical and Biomedical Engineering, City University of Hong Kong, Tae Chee Avenue Kowloon, Hong Kong 999077, China.

Center for Advanced Structural Materials, City University of Hong Kong Shenzhen Research Institute, 8 Yuexing 1st Road, Nanshan District, Shenzhen, China.

In addition, the Acknowledgements section in this Article contained errors:

"The authors gratefully acknowledge the financial support of the ITER-National Magnetic Confinement Fusion Program (2010GB109000 and 2014GB123000). J. LU acknowledges the financial support provided by the MOST 973 Program of China under the grant 2012CB932203 and the Croucher Foundation Grant (CityU9500006)."

now reads:

"The authors gratefully acknowledge the financial support of the ITER-National Magnetic Confinement Fusion Program (2010GB109000 and 2014GB123000). J. LU acknowledges the financial support from Guangdong Science and Technology Department (Ref: 2014B050504003) and SZSTI (Ref: ZDSYS201602291653165).”

These errors have now been corrected in the HTML and PDF versions of this Article.

\footnotetext{
${ }^{1}$ State Key Laboratory for GeoMechanics and Deep Underground Engineering, China University of Mining and Technology, Beijing, 100083, China. 'Institute of Nuclear Materials, University of Science \& Technology Beijing, Beijing, 100083, China. ${ }^{3}$ Department of Mechanical and Biomedical Engineering, City University of Hong Kong, Tae Chee Avenue Kowloon, Hong Kong, 999077, China. 'Laboratory for Nuclear Materials, Paul Scherrer Institute, 5323, Villigen PSI, Switzerland. ${ }^{5}$ Center for Advanced Structural Materials, City University of Hong Kong Shenzhen Research Institute, 8Yuexing 1st Road, Nanshan District, Shenzhen, China. Hong-Yan Guo and Min Xia contributed equally to this work. Correspondence and requests for materials should be addressed to M.X. (email: xmdsg@ustb.edu.cn) or J.L. (email: jianlu@cityu.edu.hk) or C.-C.G. (email: ccge@mater.ustb.edu.cn)
} 
(i) Open Access This article is licensed under a Creative Commons Attribution 4.0 International License, which permits use, sharing, adaptation, distribution and reproduction in any medium or format, as long as you give appropriate credit to the original author(s) and the source, provide a link to the Creative Commons license, and indicate if changes were made. The images or other third party material in this article are included in the article's Creative Commons license, unless indicated otherwise in a credit line to the material. If material is not included in the article's Creative Commons license and your intended use is not permitted by statutory regulation or exceeds the permitted use, you will need to obtain permission directly from the copyright holder. To view a copy of this license, visit http://creativecommons.org/licenses/by/4.0/.

(C) The Author(s) 2018 